\title{
Advocacy for horticulture!
}

More than three thousand participants met from 22nd to 27th of August 2010 in Lisbon, Portugal, during the 28th International Congress on Horticulture. They highlighted and discussed the key role that fruits and vegetables can play as an essential component of economic development, considering their nutritional value and their ability to generate income and help to reduce poverty.

Meanwhile, chronic diseases are exploding in developing countries; thus, cardiovascular diseases currently affect over 20 million people in sub-Saharan Africa and, according to WHO estimates, they could be responsible for more than $50 \%$ of deaths from all causes in 2020 if nothing is done.

Now, it is increasingly recognized that fruits and vegetables could significantly contribute to alleviating these diseases if they were consumed much more in the diet of people in these countries. But, at the same time, horticulture is virtually absent from the interna- tional agenda dealing with food security and poverty reduction, even in the mega-programs of the CGIAR, where the focus is mainly on cereals, basic food, fish and meat, which is agriculture; food-borne infections and intoxications, and water-associated, zoonotic and occupational diseases for the health side.

It is high time to take this situation into account better and advocate for horticulture as a key to more effectively achieving the millennium development goals.

The messages shared during the 28th Congress on Horticulture in Lisbon must be transmitted beyond this forum and relayed by the media and other channels to reach decision-makers, to finally become part of the international agenda for development.

Dr. Jacky Ganry Scientific Director of Fruits 


\section{Un plaidoyer pour l'horticulture !}

Plus de trois mille participants se sont réunis du 22 au 27 août 2010 à Lisbonne, au Portugal, à l'occasion du $28^{\mathrm{e}}$ Congrès International sur l'Horticulture. Ils ont souligné et discuté du rôle clé que les fruits et les légumes peuvent jouer en tant que composante essentielle du développement économique, compte tenu de leur valeur nutritive et de leur capacité à générer des revenus, contribuant ainsi à réduire la pauvreté.

Pendant ce temps, les maladies chroniques explosent dans des pays en développement; les maladies cardio-vasculaires affectent ainsi, actuellement, plus de 20 millions de personnes en Afrique subsaharienne et, selon les prévisions de l'OMS, elles pourraient être responsables de plus de $50 \%$ des décès, toutes causes confondues, en 2020, si rien n'était fait.

Or il est de plus en plus reconnu que les fruits et légumes pourraient fortement contribuer à atténuer ces maladies, s'ils étaient beaucoup plus présents dans l'alimentation des populations de ces pays. Mais, en même temps, l'horticulture est pratiquement absente de l'agenda international traitant de la sécurité alimen- taire et de la réduction de la pauvreté, cela même dans les méga-programmes du GCRAI où l'accent est surtout mis sur les céréales, les aliments de base, le poisson et la viande pour ce qui est de l'agriculture ; les infections et intoxications d'origine alimentaire et associées à l'eau, les maladies zoonotiques et professionnelles pour le côté santé.

Il est grand temps de mieux prendre en compte cette situation et de plaider en faveur de l'horticulture comme un élément clé apte à atteindre plus efficacement les objectifs de développement du millénaire.

Les messages partagés au cours du $28^{\mathrm{e}}$ Congrès Horticole de Lisbonne doivent être transmis au-delà de ce forum et relayés par les médias et d'autres canaux pour atteindre les décideurs afin de faire enfin partie de l'agenda international traitant du développement.

Dr. Jacky Ganry Directeur scientifique de Fruits 